\title{
Estudio Socioeconómico de un Proyecto de Reforestación Implementado con Pequeños Productores de Cacao en Honduras
}

\author{
Karent Kupferschmied ${ }^{1}$, Ingrid Fromm ${ }^{2}$ y Arthur Rouanet ${ }^{3}$
}

Resumen. El conocimiento general acerca del cultivo del cacao, las características genéticas del cacao hondureño y las condiciones agroclimáticas del país han impulsado el interés de varias entidades y países a fomentar la producción del cacao orgánico. En octubre del 2014, la Fundación Hondureña de Investigación Agrícola (FHIA) registró 4,463 ha de cacao en Honduras. En busca de alternativas ambiental y económicamente amigables, desde el 2009, la Fundación Helvetas consolidó la Asociación de Productores de Sistemas Agroforestales con cacao orgánico de Olancho (APROSACAO) y con la ayuda de la inversión privada de Chocolats Halba y Pur Projet, en el 2012. Se inició el programa de reforestación para aumentar la captura de carbono y asegurar los ingresos a futuro de los productores, mediante la comercialización de madera legalizada. El objetivo del estudio fue determinar el impacto socioeconómico del programa de reforestación de APROSACAO/ Pur Projet y para esto se muestrearon 55 productores en cinco veredas en Olancho, Honduras. En el aspecto económico no se observa, hasta el momento, un efecto directo en el ingreso o en el aumento de la productividad del cacao. No obstante, los productores tienen mayores ingresos que gastos. Además, hubo una influencia positiva del programa de reforestación en términos de suficiencia e independencia alimentaria y a su contribución al medio ambiente y futuras generaciones.

Palabras clave: Cacao orgánico, cooperativa, Olancho, sistemas agroforestales.

\section{Socio-Economic Study of an Implemented Agroforestry Project with Small Scale Cocoa Producers in Honduras}

\begin{abstract}
The general knowledge about cocoa, the genetic traits of Honduran cocoa, and the particular environmental conditions of the country has been driving the interest of several organizations and countries to encourage sustainable cocoa production. In October 2014, the Fundación Hondureña de Investigación Agrícola (FHIA) reported 4,463 ha of cocoa planted nationwide. Seeking for environmental and economic alternatives, Helvetas Swiss Intercooperation has strengthened since 2009 the Honduran Association of Organic Cocoa Producers from Olancho (APROSACAO) with private investment from Chocolats Halba Coop Group and Pur Projet since 2012. The reforestation program was initiated in order to increase carbon sequestration and ensure the future producers' revenues through the marketing of legal wood. The objective of the study was to identify the socio economic impact of the agroforestry program APROSACAO/Pur Projet among cocoa producers. For the purpose of the study, data from 55 producers living in 5 villages in Olancho was collected. The results indicated that there is no direct effect in the income or cocoa yield improvement. Nonetheless, cocoa producers report more income than expenses. In addition, there is a positive influence of the agroforestry program on food security and cocoa producers' contribution to the environment and future generations.
\end{abstract}

Keywords: Organic cocoa, Agroforestry systems, Olancho, Cooperative.

\section{Introducción}

El cultivo de cacao ha sido de gran tradición en
Honduras y de la región mesoamericana. Durante la década entre 1980 y 1990, el sector cacaotero en Honduras tuvo un importante auge debido a que los

\footnotetext{
${ }^{1}$ Universidad de Ciencias Aplicadas de Berna (BAF). Länggasse 85, 3032 Zollikofen, Berna, Suiza. Correo electrónico Karent.kupferschmied@gmail.com

${ }^{2}$ Universidad de Ciencias Aplicadas de Berna (BAF). Länggasse 85, 3032 Zollikofen, Berna, Suiza. Correo electrónico ingrid.fromm@bfh.ch

${ }^{3}$ Pur Projet. 4 Rue de la Pierre Levée, 75011 Paris, Francia. Correo electrónico arthur@pureprojet.com
} 
precios internacionales eran altos (Fromm 2014). Sin embargo, la mayor parte de las plantaciones de cacao fueron afectadas con el paso del huracán Mitch en 1998, causando pérdidas económicas para los productores (Fromm 2014). Lo anterior, sumado a procesos de migración, cambió las actividades económicas (Vallejo 2011), aumentó la ganadería y con ello acelerando la tasa total anual promedio de deforestación, que de acuerdo con Murillo (2011) para el periodo del 2001 al 2005, en una de las áreas protegidas del país como lo es el Parque Nacional Patuca, se encontraba en 13,737.3 ha/año.

A pesar de la importancia de los bosques y de los productos forestales no maderables, no solo para el consumo interno sino para el mercado internacional, hasta hace unos 7 años han sido valorados y recibido el apoyo institucional a través de convenios de cooperación internacional y proyectos de reforestación asociados a las actividades agrícolas en el país (FHIA 2015b). Un ejemplo es la promoción de programas de reforestación asociados al cultivo del cacao.

Según la Secretaría de Agricultura y Ganadería (SAG), hasta octubre del 2014 habían 4,463 ha de cacao en Honduras (FHIA 2015b). El área promedio de producción es menor a 2.0 ha (pequeños productores), se encuentra arriba de los $200 \mathrm{msnm}$ y está asociada a sistemas agroforestales.

El apoyo de entidades públicas y privadas a las organizaciones de pequeños productores incluye inversiones de capital humano (principalmente capacitaciones), material vegetal (de cacao y árboles frutales) e infraestructura (instalaciones de poscosecha); ha mejorado la cadena de valor del cacao y permitido el desarrollo de la agricultura por contrato, asegurando un precio mínimo de compra favorable para los agricultores (Sánchez et al. 2015).

La producción de madera y utilización de productos forestales no maderables del bosque representan una fuente de diversificación de alimentos para consumo y/o venta en los mercados y provisión de materiales de construcción; que finalmente contribuyen a: mejorar la calidad de vida de las familias cacaoteras en la seguridad alimentaria e ingresos extra, al medio ambiente gracias al incremento en sumideros de carbono y finalmente a la motivación de las próximas generaciones a seguir produciendo cacao (Girade 2013).
El conocimiento acerca del cultivo, las características genéticas del cacao que se produce internamente (resistencia a enfermedades, aroma y sabor superior) y las condiciones agroclimáticas favorables para explotar el cultivo ha impulsado el interés de entidades y países a fomentar la producción del cacao orgánico (Chavez y Chafla 2009). La Fundación Hondureña de Investigación Agrícola (FHIA) ha sido clave en fomentar los Sistemas Agroforestales (SAF), a través de proyectos como: Promoción de Sistemas Agroforestales de Alto Valor con Cacao en Honduras, que inició en abril de 2010 con la firma del convenio respaldado por The Canadian International Development Agency (CIDA).

En el departamento de Olancho, Honduras, zona de importancia por ser la zona amortiguadora del Parque Nacional Patuca, desde el 2009 se ha fomentado el mejoramiento de las condiciones de vida de los productores de cacao mediante la consolidación de la Asociación de Productores de Sistemas Agroforestales con cacao orgánico de Olancho (APROSACAO). Gracias a la cooperación entre la Fundación Helvetas Swiss Intercooperation, la compañía suiza Chocolats Halba- Coop Group y el apoyo de la empresa Pur Projet, se han capacitado a los productores en la cadena de valor del cacao e incentivado la sostenibilidad ambiental, a través de reforestación, teniendo como objetivo la plantación de árboles en consorcio con cacao y la creación de zonas silvopastoriles (Hernández 2015).

Pese a que el asocio y uso de SAF en cacao se ha extendido en Honduras, en Olancho se conoce y fomenta hasta hace poco. Además, no existe literatura referente a la evaluación de APROSACAO y su gestión en las veredas, durante sus años de funcionamiento en Olancho. Es por esto que se ha visto la necesidad de evaluar el estado de satisfacción de los productores de cacao con su organización (APROSACAO), la implementación o puesta en marcha del programa de reforestación y con la actividad de producción en general en el departamento de Olancho, Honduras.

\section{Materiales y Métodos}

La investigación de campo duró del 2 de julio al 3 de septiembre del 2015. Una metodología descriptiva fue usada con énfasis en comparar los productores agrícolas (cultivos de cacao u otros cultivos) 
pertenecientes a la APROSACAO en lo que se refiere a evaluar las problemáticas de las aldeas, el estado de los productores (frecuencia de participación por género, ingresos o principal actividad económica), grado de satisfacción con su organización, nivel de participación en el programa de reforestación y enfoque futuro de ayudas de la APROSACAO (de acuerdo a lo esperado por el productor).

Se encuestaron 55 productores en las cinco veredas, únicas zonas de acción del programa de reforestación de la APROSACAO/Pur Projet, denominadas Cuyamel, Poncaya, Río Blanco, Río Tinto y Catacamas del departamento de Olancho, Honduras, que es el $14 \%$ de la población de interés (Figura 1). El muestreo fue de efecto multiplicador porque gran parte de la investigación fue cualitativa y con entrevistas. "Este método se basa en el supuesto que existe un vínculo entre la muestra inicial y otros en la misma población objetivo, permitiendo tomar ventaja de la red social de los entrevistados para proporcionar a un conjunto cada vez mayor de contactos potenciales" (Atkinson y Flint 2001).

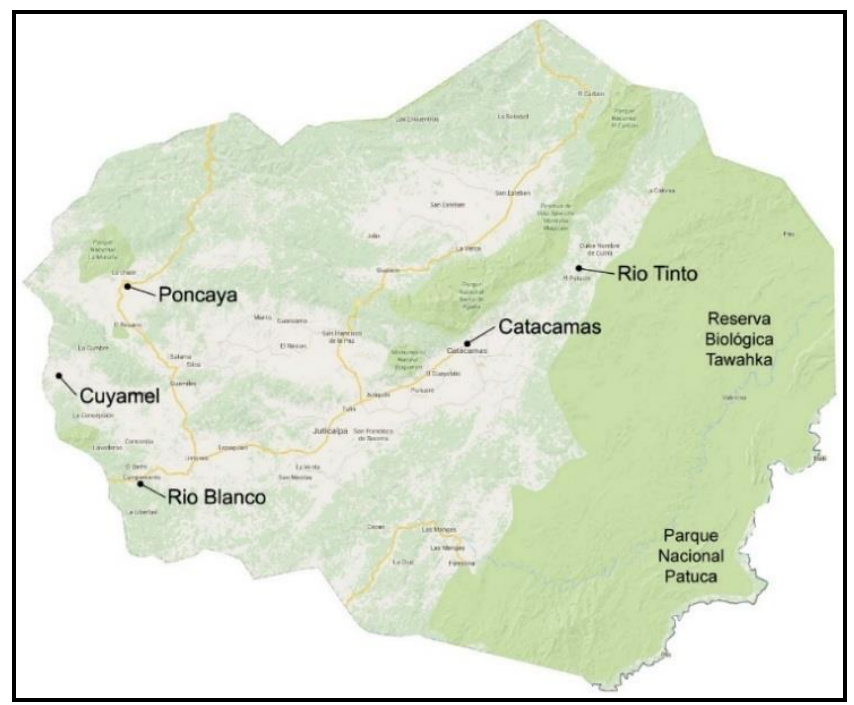

Figura 1. Departamento de Olancho, Honduras, y las cinco zonas de acción o veredas del programa de reforestación de APROSACAO/Pur Projet.

Análisis estadístico. Se usó una metodología descriptiva con énfasis en comparar los productores agrícolas (cultivos de cacao $u$ otros cultivos) pertenecientes a la APROSACAO. Los datos obtenidos fueron analizados y los resultados interpretados usando el software estadístico Rstudio mediante Análisis de Varianza (ANDEVA), prueba de Kruskall-Wallis y coeficiente de regresión, para determinar la correlación entre el rendimiento del cacao en baba con el ingreso doméstico mensual de los productores de la APROSACAO para estimar la diferencia en las fuentes de ingreso en las zonas.

\section{Resultados}

Perfil de los productores encuestados. Es evidente que en la cadena de valor del cacao en Honduras hay bajos niveles de tecnología en la producción y poscosecha, falta de conocimiento de los precios de mercado, envejecimiento de los agricultores que dependen del cacao para su subsistencia y alto índice de analfabetismo. Los proyectos realizados a través de la cooperación entre organizaciones en la promoción de SAF han fortalecido la cadena de valor del cacao. Las familias productoras han aumentado sus ingresos debido al incremento de precio y hay una clara tendencia de aumento de volumen de cacao que se beneficia y exporta para el mercado de alta calidad (FHIA 2015a).

El $85 \%$ de los productores son hombres y $15 \%$ mujeres, el rango de edad va de 19 hasta 84 años (Cuadro 1), tienen un nivel de alfabetización básico, pues solo el $22 \%$ de los productores encuestados no cuenta con ningún grado de instrucción; y todos ellos son de pequeña escala. El promedio del área para la producción agrícola es 4.0 ha, que se divide en 1.44 ha para el cultivo de cacao y 1.56 ha para otros cultivos. Dichos valores son mayores en los departamentos de Cortés y Yoro en Honduras, en donde el tamaño de las fincas destinadas a la asociación de cacao con SAFs es de 2.41 en promedio (Martínez 2011).

Solo 20 de los 55 productores han cosechado cacao en baba, los que no han cosechado pertenecen en su mayoría a las veredas o zonas de Catacamas y Cuyamel. En esta zona, el primer pico de cosecha es entre 3 y 4 años (dependiendo de la variedad) y alrededor de los 6-7 años, el cacao se cosecha quincenalmente. Río Blanco es la zona que tiene mayor promedio de área de producción (2.14 ha) y consecuentemente mayor rendimiento de cacao 
en baba (337 kg/mensuales); la edad del cultivo oscila entre 4-5 años. En Honduras "se han hecho estimaciones de producción considerando la edad de las plantaciones y el manejo que le den los productores a la mismas, en fincas rehabilitadas se estima conservadoramente una producción de cacao de $300 \mathrm{~kg} / \mathrm{ha}$ el primer año y de $600 \mathrm{~kg} / \mathrm{ha}$ a partir del segundo año. En el caso de fincas nuevas, se estima un rendimiento de $300 \mathrm{~kg} / \mathrm{ha}$ en el tercer año de edad, $400 \mathrm{~kg} / \mathrm{ha}$ en el cuarto, $600 \mathrm{~kg} / \mathrm{ha}$ en el quinto y $700 \mathrm{~kg} / \mathrm{ha}$ es a partir del sexto año" (FHIA 2015b).

Cuadro 1. Detalle de los 55 productores encuestados pertenecientes a la Asociación de Productores de Sistemas Agroforestales con cacao orgánico de Olancho (APROSACAO).

\begin{tabular}{|c|c|c|c|c|c|c|c|c|c|}
\hline \multirow[b]{2}{*}{ Variable } & \multirow[b]{2}{*}{$\begin{array}{l}\text { Edad } \\
\text { (años) }\end{array}$} & \multicolumn{2}{|c|}{$\begin{array}{l}\text { Lempiras } \\
\text { mensuales }\end{array}$} & \multicolumn{2}{|c|}{$\begin{array}{l}\text { Hectáreas en } \\
\text { producción }\end{array}$} & \multirow[b]{2}{*}{$\begin{array}{l}\text { Variedades } \\
\text { cultivadas }\end{array}$} & \multicolumn{2}{|c|}{$\begin{array}{c}\text { Rendimiento } \\
\text { mensual de cacao } \\
\text { en baba por } \\
\text { hectárea }(\mathrm{kg})\end{array}$} & \multirow{2}{*}{$\begin{array}{c}\text { Años } \\
\text { produciendo } \\
\text { cacao }\end{array}$} \\
\hline & & Ingreso & Gasto & Cacao & $\begin{array}{c}\text { Otros } \\
\text { cultivos }\end{array}$ & & Real & Esperado & \\
\hline Media & 40.5 & 8,228 & 6,101 & 1.44 & 1.56 & 1.72 & 108.84 & 4.56 & 2.66 \\
\hline $\begin{array}{l}\text { Desviación } \\
\text { estándar }\end{array}$ & 15.4 & 10,581 & 7,179 & 1.69 & 3.79 & 3.60 & 225.34 & 8.15 & 1.45 \\
\hline $\begin{array}{l}\text { Valor } \\
\text { mínimo }\end{array}$ & 19.0 & 1,200 & 600 & 0.00 & 0.00 & 0.00 & 0.00 & 10.00 & 0.00 \\
\hline $\begin{array}{l}\text { Valor } \\
\text { máximo }\end{array}$ & 84.0 & 48,000 & 40,030 & 12.00 & 28.00 & 27.00 & 800.00 & 4200.00 & 6.00 \\
\hline
\end{tabular}

Se observa una clara diferenciación y división de trabajo de acuerdo al género. En la cadena de valor del cacao, los hombres trabajan en el eslabón de producción, mientras que las mujeres se encargan de las actividades de transformación y procesamiento de los granos de cacao. Gracias a las capacitaciones ofrecidas por la APROSACAO con miras al empoderamiento femenino, muchas de ellas hacen parte de la microempresa chocolatera Chocolan dedicada a la producción de chocolates en barra, cacao en polvo y pinol, destinado al mercado local como pulperías y pequeños supermercados.

Seguridad financiera. Para evaluar este aspecto, se tomó en cuenta la percepción del ingreso bruto en los pasados 2 años basado en la comparación de otras familias en la comunidad y la capacidad de suplir las necesidades domésticas. El $55 \%$ de los productores afirma que su ingreso ha mejorado, si se compara lo que ganaba hace 2 años, el $35 \%$ que se ha mantenido, el $4 \%$ que ha desmejorado y un $4 \%$ dice no tener conocimiento. Esta mejoría posiblemente se debe a que el asocio con sistemas forestales les permite a los productores tener ingresos a corto, mediano y largo plazo por la venta de las cosechas. Desafortunadamente, el $78 \%$ de los productores asegura la insuficiencia de sus ingresos para cubrir las necesidades de su familia.

Servicios públicos. Todos los productores encuestados cuentan al menos con una fuente hídrica para suplir las necesidades domésticas. El recurso hídrico más común son las quebradas, las cuales abastecen al 35\% de la población encuestada, aunque solo el $27 \%$ de los productores reportó tener acceso al servicio de agua potable. Lo anterior, debido a que Catacamas, hasta agosto del 2015, fue la única zona con un proyecto de tratamiento de agua. Solamente el $65 \%$ de los productores tiene la posibilidad de contar con una fuente eléctrica.

Calidad de vida de los productores de la APROSACAO. En promedio, el gasto doméstico mensual para el periodo de enero a agosto del 2015 se estimó en L. 6102; este valor varía de acuerdo al número de personas en el hogar. Un productor necesita disponer como mínimo L. 600 por persona para cubrir sus necesidades mensuales. Si bien la 
mayor parte de los productores no devenga ningún salario o entrada propia de la venta del cacao (puesto que no ha empezado el ciclo de producción), muchos de los productores reciben remesas de sus hijos $u$ otros parientes que se encuentran trabajando en Tegucigalpa o incluso en el exterior, o de la venta de víveres en las pulperías (en el caso de las mujeres) como principal fuente económica. De acuerdo al estudio de Charles (2007:9), "En Honduras las remesas contribuyen con un importante porcentaje del Producto Interno Bruto (PIB) del país, pues son destinadas al $81 \%$ de los hogares a nivel nacional, dentro del cual el $48 \%$ se encuentran en la zona rural". "En el 2006, los emigrantes hondureños enviaron más de US\$2359 millones a Honduras y por lo tanto estas pueden ser un potencial para el desarrollo del país" (Charles 2007:13). Las mujeres son las principales receptoras de remesas (52\%) y los principales beneficiarios son los hermanos/as, hijos/as, padres y cónyuges, quienes obtienen L. 105 más en su ingreso per cápita, si se compara con los hogares no receptores de remesas. Finalmente, los hogares beneficiarios de remesas gastan la mayor parte en necesidades básicas y es poco lo que destinan para educación, inversión en propiedades y pequeños negocios familiares (Charles 2007:14). Adicionalmente, se calculó la existencia de una correlación entre el ingreso doméstico mensual y el rendimiento del cacao en baba por medio de una regresión linear (Figura 2). El resultado del análisis nos da la ecuación:

$$
y=6783.937+6.993 x
$$

Por lo que se deduce que el promedio del ingreso doméstico mensual incrementa en $6.993 \mathrm{~L} / \mathrm{kg}$ de baba de cacao producido, si se considera que la producción de cacao en baba mensual oscila entre 0 $\mathrm{kg}$ y $800 \mathrm{~kg}$. Igualmente, el test calcula un coeficiente de determinación $\mathrm{R}^{2}=0.02764$ y un valor de $p$ de 0.2641 , indicando que el rendimiento del cacao no tiene ningún efecto en el ingreso doméstico mensual en las regiones evaluadas. Lo anterior se podría deber a que los cacaotales son jóvenes y para el periodo de evaluación todavía no habían entrado a su etapa productiva.

Conocimiento local acerca del cacao. El tiempo de producción de cacao de los productores afiliados a la APROSACAO varía desde unos meses hasta 6 años, con un promedio de 3 años. La mayor parte de los productores tiene cultivos de cacao jóvenes con material vegetal híbrido mejor conocido como trinitario. Sin embargo, algunos tienen materiales criollos de alta calidad, aroma y por ende mayor precio en el mercado. Con la encuesta se pudo inferir que la principal problemática con el material genético criollo es la falta de conocimiento de los productores frente al potencial de mercado de estos granos, y en campo la baja producción de frutos y alta inversión de trabajo en podas estructurales y de mantenimiento, razón por la que muchos de ellos terminan erradicándolos de su predio.

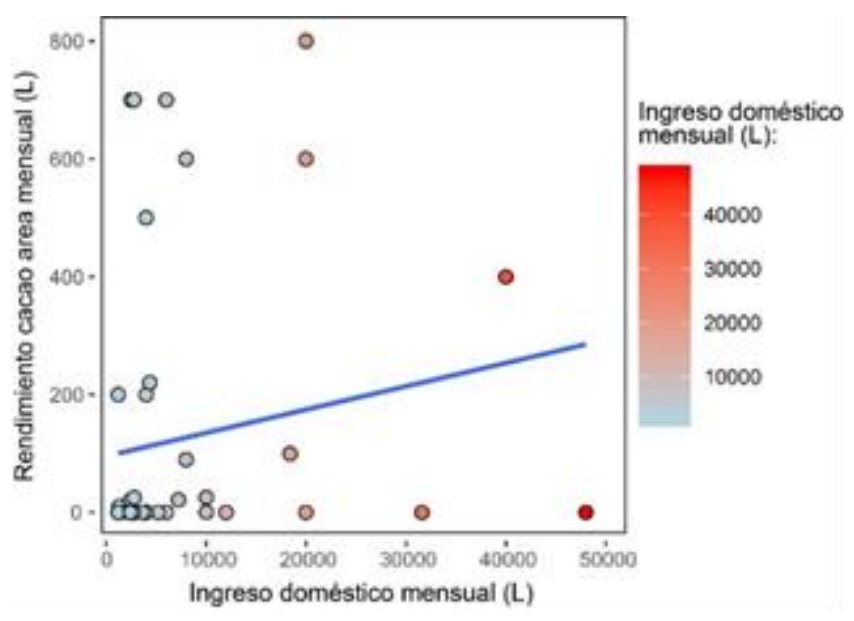

Figura 2. Correlación entre el rendimiento de cacao en baba mensual y el ingreso doméstico mensual (L.) de las zonas de acción de la Asociación de Productores de Sistemas Agroforestales con cacao orgánico de Olancho (APROSACAO).

SAF. El $89 \%$ de los productores pertenece al programa de reforestación de la APROSACAO/Pur Projet, todos ellos como se mencionó anteriormente cacaocultores. Pese a que el programa de reforestación tiene cuatro modelos a disposición, la media de uso es de 1.5 modelos por productor; o en otras palabras, el $60 \%$ de los productores maneja un modelo por predio, el $32 \%$ dos, el $2 \%$ tres y el $4 \%$ remanente, la totalidad de los modelos (Figura 3). Lo anterior en relación al tamaño del predio, interés del productor y posibilidad de acceso (tiempo de establecimiento, facilidad de asesoría o material de siembra y mano de obra disponible). 
En el 2015, el programa de reforestación estimó plantar de 8,000 a 10,000 árboles frutales (mangos, guayaba, naranjos y aguacates) en los cuatro modelos propuestos por el programa de la APROSACAO/Pur Projet y la evaluación de almacenamiento de carbono en los árboles del proyecto está en curso" (Hernández 2015).

Concerniente al estado de satisfacción de los productores pertenecientes a la APROSACAO/Pur Projet, el $83 \%$ de los encuestados dice sentirse bien y motivado a seguir colaborando con el medio ambiente, mientras las condiciones de ayuda se sigan manteniendo. De acuerdo con los productores afiliados al programa de reforestación de la APROSACAO/Pur Projet el mayor limitante para los SAFs ha sido el manejo de plagas y enfermedades con el $69 \%$ de las respuestas, seguido por sequía con el $34 \%$ de las respuestas (Figura 4). Sin embargo, estas dos problemáticas no se relacionan mutuamente.

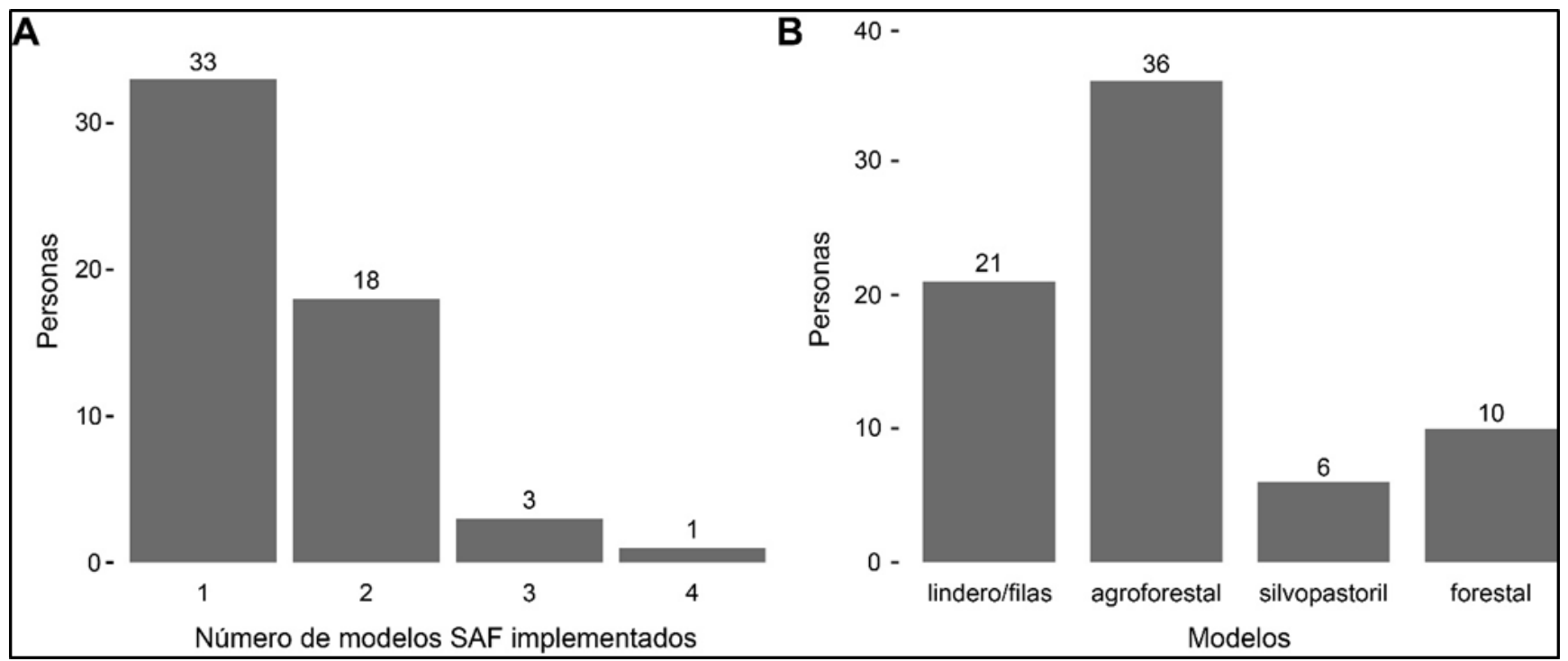

Figura 3. A) Frecuencia del uso de modelos de sistemas agroforestales (SAF) usado por los productores del programa de reforestación de la Asociación de Productores de Sistemas Agroforestales con Cacao Orgánico de Olancho (APROSACAO/Pur Projet). Los números en la parte superior de las barras muestran la cantidad de personas que respondió tener cada modelo. B) Frecuencia del uso de acuerdo al tipo de modelo SAF. Los números en la parte superior de las barras muestran el número de personas que respondió tener cada modelo.

La plaga comúnmente encontrada es el taladrador de las meliáceas o barrenador de yemas (Hypsipyla grandella (Zeller)), el cual taladra los brotes de los árboles de la familia Meliaceae, especialmente las caobas y los cedros. Los taladradores de las caobas atacan brotes nuevos, generando los mayores daños, en vivero y plantaciones jóvenes (Howard y Merida 2014). Desafortunadamente, según (Howard y Merida 2014), en los trópicos, "el taladrador de las meliáceas está activo todo el año, con la actividad concentrada en la temporada de lluvias, la cual es el período de crecimiento de brotes de las caobas" y es un insecto de difícil control pues hasta las poblaciones ligeras pueden causar daños significativos.

En el caso de la sequía, en agosto del 2015 en el departamento de Olancho, la lluvia no superó los 30 $\mathrm{mm}$ y la temperatura máxima registrada en la estación meteorológica de Catacamas fue $34^{\circ} \mathrm{C}$. Lo que dio como resultado un déficit de lluvia en más de 
$60 \%$, si se compara con el resto del territorio hondureño (Servicio Meteorológico Nacional Honduras 2015).

Manejo y dosel de sombra. Para esta sección de la encuesta se les preguntó a los productores que tipo de árboles tenían asociados en su cultivo de cacao, y se determinó la frecuencia de especies maderables y frutales. En general, hay una tendencia en la preferencia de especies maderables frente a las frutales (Figura 5). Entre los maderables tienen especies de alto valor comercial como caoba, laurel y cedro; y en los frutales las musáceas, guama y aguacate. Como la pregunta es abierta con múltiple opción de respuesta, los productores generalmente mencionaron más de una especie de árbol asociada al cultivo del cacao. El $82 \%$ de los productores pertenecientes al programa de reforestación dijo tener caoba (Swietenia macrophylla) como especie maderable en su sistema agroforestal, el 53\% laurel (Cordia alliodora), el $47 \%$ cedro (Cedrela odorata), el $22 \%$ teca (Tectona grandis), el $7 \%$ carreto (Samanea saman) y el $2 \%$ granadillo (Dalbergia tucurensis). Las especies más comunes de frutales fueron las musáceas (Musa spp.) 38\%, el guamo (Inga edulis) $36 \%$, el aguacate (Persea americana) 32\%, el mango (Mangifera indica) $10 \%$ y los cítricos (Citrus spp.) $10 \%$.

En Honduras, Martínez (2011) estudió el aporte de los sistemas agroforestales con cacao en cinco municipios de los departamentos de Cortés y Yoro, reportando la presencia de árboles como caoba, cedro, laurel, hormigo (Platymiscium dimorphandrum) y San Juan (Cupressus lindleyi) de alto valor económico y usadas para madera. Así como una alta frecuencia ( $88 \%$ de las fincas) de madriago (Gliricidia sepium) y laurel blanco (Cordia alliodora) "la primera que por tradición se ha utilizado como sombra permanente en cacao y la segunda por el valor de la madera" (Martínez 2011: 42); y de árboles frutales como el mango, aguacate y las musáceas (especialmente el plátano).

Las musáceas son de importancia para Honduras, pero el verdadero valor es para aquellas familias de las zonas rurales que las usan para devengar ingresos y para consumo propio (Martínez 2011: 29). Siguiendo a Torres (2009), las demás especies frutales mencionadas por los productores de la APROSACAO, como el guamo, el aguacate, el mango y los cítricos, son cultivadas debido al acceso gratuito de estas en el programa de reforestación. Las primeras dos mencionadas son requeridas por los productores, por su alta estabilidad de precio de venta en el mercado local; el mango y los cítricos por su demanda (autoconsumo y mercado local).

En el uso de los maderables, tal como era de esperarse, el $51 \%$ de los productores espera comercializar la madera y así generar ingresos extra, el $45 \%$ dice que el uso de los árboles radica en la provisión de alimentos, el $2 \%$ considera que los árboles prestan servicios ecológicos como generadores de barreras rompevientos y el $2 \%$ remanente como sombrío necesario para el establecimiento y desarrollo del cacao.

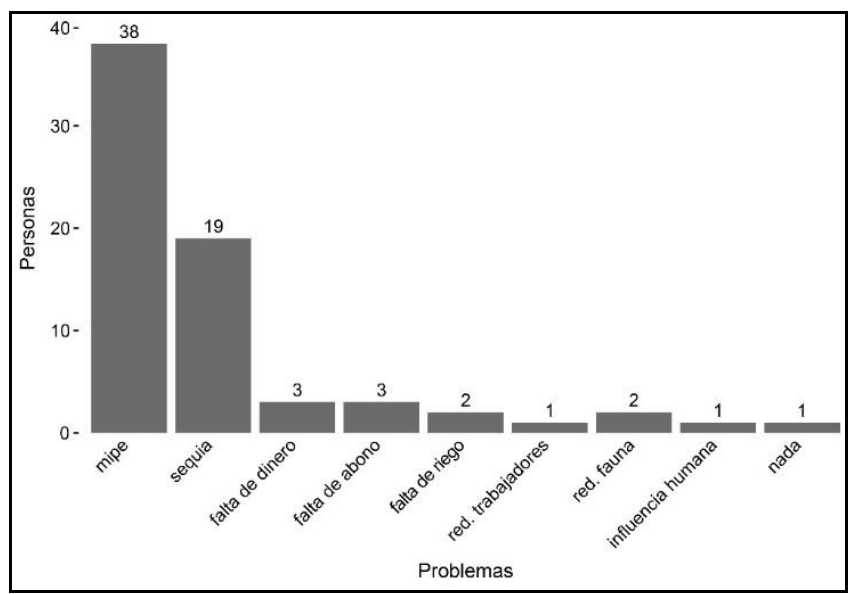

Figura 4. Frecuencia de los problemas que afectan los SAF. Si bien la encuesta incluyo 55 personas, la pregunta fue de selección múltiple. Los números en la parte superior de las barras muestran el número de personas que respondió cada problema. Abreviaciones/observaciones: mipe: manejo de plagas y enfermedades; red. trabajadores, reducción de trabajadores en la zona; red. fauna: reducción de fauna; influencia humana, referente a robo de frutos.

Intervención de la APROSACAO. En Olancho, la intervención de las entidades ha sido mínima, es por esto que la APROSACAO tiene relevancia no solo en la zona, sino en el fortalecimiento de la cadena del valor del cacao y pese a que el tiempo de gestión es relativamente corto, es una organización de los productores y esta para su servicio. Romero (2014: 
21) reporta "En los últimos 4 años se ha registrado un aumento del número de organizaciones con capacidades para acopiar, secar y fermentar el cacao, esto se debe a que las organizaciones ya tienen contratos de compra con Chocolats Halba y las nuevas áreas ya están produciendo". En Olancho, la APROSACAO, como organización, genera confianza entre los productores. Es imprescindible para ellos el respaldo de organizaciones internacionales y empresas privadas en los proyectos que se desarrollan, pues genera confianza en el uso de los recursos y mecanismos de vigilancia y control. De acuerdo con Romero (2014: 22) "históricamente el cacao comercializado en el país ha tenido una calidad baja, ya que los estándares pedidos por los compradores y los mercados en que se comercializaban no demandaban calidad sino volumen. Por lo que el rol de la empresa privada ha sido clave en la promoción de prácticas sostenibles para la producción de cacao".

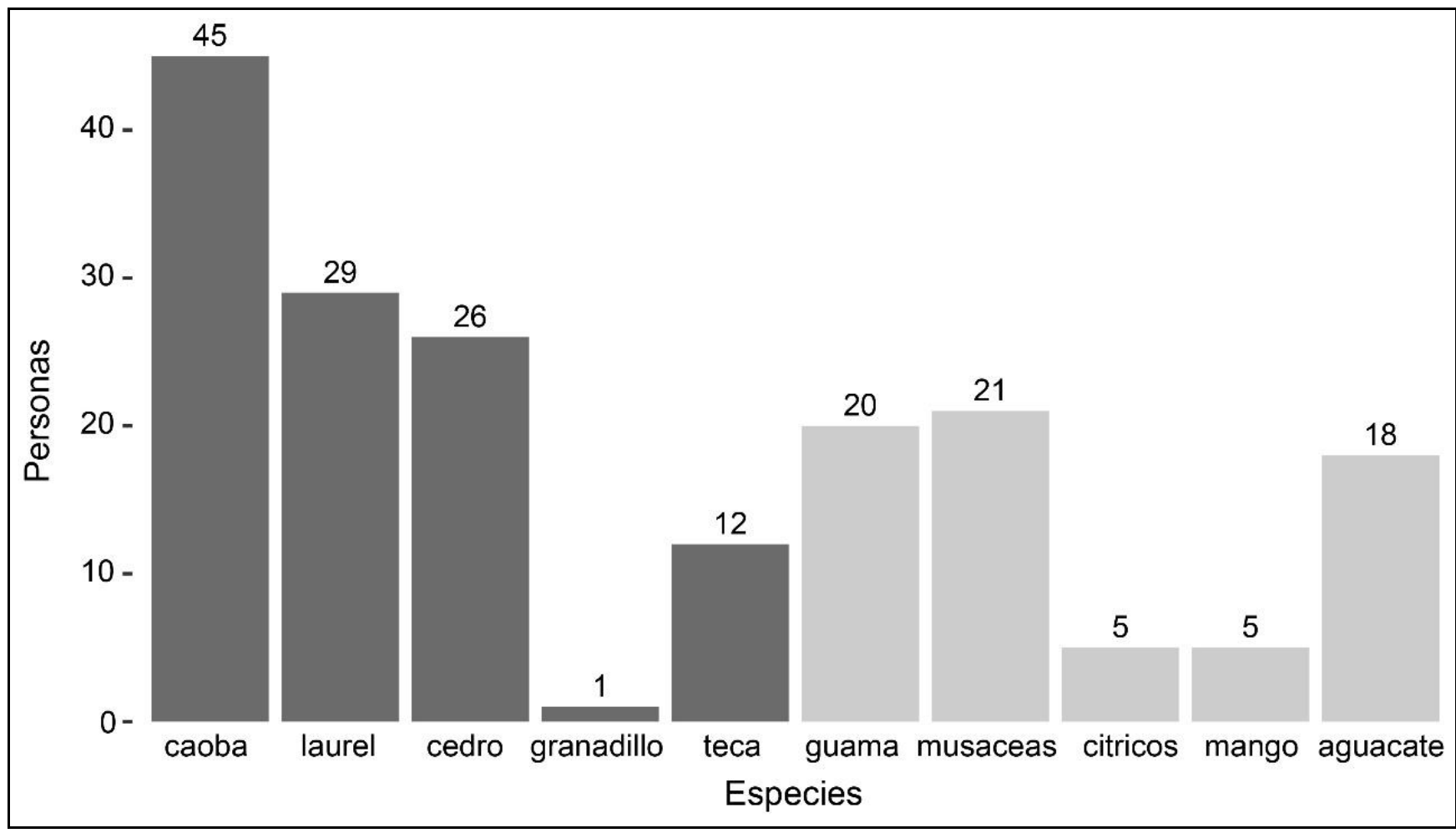

Figura 5. Frecuencia de especies vegetales asociadas a los SAF. Las especies maderables están representadas en color gris oscuro y las especies frutales en color gris claro. Los números en la parte superior de las barras muestran el número de productores que mencionó disponer de cada especie vegetal en su predio.

El $62 \%$ de los productores tienen entre 2 a 3 años de participar en la organización, con lo que se infiere que sus plantaciones son jóvenes y probablemente están próximas a dar su primera cosecha; el $31 \%$ más de 3 años, de hecho muchos de ellos empezaron cuando la APROSACAO dio inicio y por ende son cacaocultores con mucha experiencia y conocimiento acerca del cultivo y probablemente con cosechas quincenales; y el $7 \%$ restante son socios que hasta ahora empiezan a cultivar cacao con la APROSACAO y están a la espera de su primera cosecha. 
Una vez se considera el tiempo de participación, es importante para la organización saber los motivos que impulsaron a los productores a ser socios de la APROSACAO para así fortalecer esta ventaja o fomentar oportunidades para atraer más productores.

Entre los resultados expuestos (Figura 6), la mayor parte de los productores $(29 \%)$ dijeron acudir por curiosidad; el $20 \%$ mencionó las ayudas sociales, refiriéndose a los incentivos propuestos por la plantación de maderables, los bonos, las capacitaciones y la entrega gratuita de material vegetal; el $17 \%$ dijo tener interés en la posibilidad de exportación o mercado del cacao sin utilizar varios intermediarios, el $16 \%$ la oportunidad de tener asistencia técnica para sus cultivos, el $11 \%$ el precio de compra del cacao en baba y como nuevo elemento $7 \%$ de los productores propusieron como factor extra la contribución de ellos al medio ambiente.

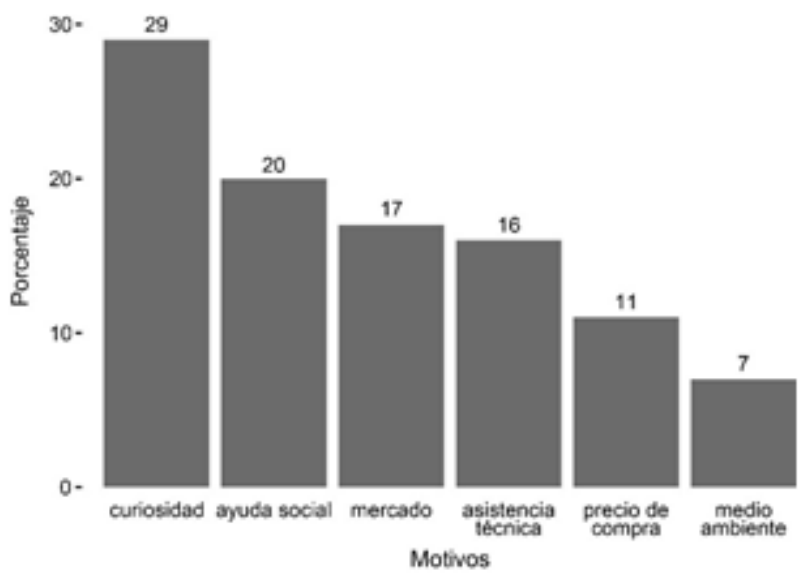

Figura 6. Motivos que llevaron a los productores a participar en la Asociación de Productores de Sistemas Agroforestales con cacao orgánico de Olancho (APROSACAO). Los números en la parte superior de las barras muestran el número de personas que respondió cada factor.

En cuanto a los compromisos de la APROSACAO y sus funcionarios con los socios, el $80 \%$ de los productores dice recibir informes de actividades y resultados de la organización y el $96 \%$ de los socios está conforme con la asistencia técnica en campo (bien por los ingenieros o por el facilitador) y las recomendaciones hechas por los ingenieros. Al evaluar la Inclusión de los productores de la APROSACAO en los procesos decisivos, el $91 \%$ de los productores sienten que la APROSACAO es su propia organización pues consideran que son parte de la toma de decisiones que se discuten en las reuniones y reportan haber obtenido muy buenos y buenos resultados con la organización.

Para finalizar la encuesta se les preguntó a los productores en que les gustaría que se enfocara la intervención de los proyectos o programas futuros de la APROSACAO. Los resultados dan a conocer la importancia en la formulación de proyectos destinados a créditos blandos, de acuerdo con el $26 \%$ de la población encuestada. Es por esto que desde el 2015, la APROSACAO ha gestionado con la dirección de registros y catastro del IP (Instituto de la Propiedad) la inscripción de predios y el otorgamiento de títulos de propiedad. La finalidad es llevar a los funcionarios a las zonas para que ellos brinden orientación, evitando estafas y reduciendo tiempo y dinero de los interesados y de la institución. La adquisición de este título les permitirá a muchos productores acceder a sistemas de financiamiento a largo plazo, sin embargo este proceso demora, requiere de la disposición de los funcionarios y de la voluntad de los productores.

El segundo aspecto escogido fueron las ayudas que le pueda ofrecer la APROSACAO al productor en compra de insumos, material de poda y siembra, sistemas de riego, aumento de injertos de cacao y ampliación de las áreas cacaoteras y/o maderables, que comprendieron el $22 \%$ de las respuestas. Si bien esto sería de gran alivio para ellos, es poco probable financiar la totalidad de los productores aún más cuando las necesidades varían de productor a productor. Lo recomendable seria abastecer cada vereda al menos con un equipo de poda e insumos.

Existe incertidumbre entre los productores en el ingreso que obtendrán de la venta de maderables, muchos son conscientes que la inversión que hacen hoy no la verán ellos, pero sí las próximas generaciones. Por lo tanto, es imprescindible un estudio (por ejemplo un flujo de caja y 30 años, tiempo en el cual la caoba del atlántico ha llegado a su debida madurez comercial) que determine si la estimación de Pur Projet a largo plazo $(11,000$ dólares por año por familia para el 2055) derivados de la venta de madera se acerca o no a la realidad. 


\section{Conclusiones}

En el presente estudio, los autores no observaron hasta el momento un efecto directo en el ingreso o en el aumento de la productividad del cacao, debido a que los cacaotales aún no han entrado a la etapa productiva. No obstante, se puede deducir del estudio que en promedio los productores tienen mayores ingresos que gastos (en parte debido a las remesas recibidas), sin embargo estos valores están en función del número de personas por hogar que aportan o no a la sostenibilidad familiar. Igualmente, se determinó una influencia positiva del programa de reforestación a nivel de suficiencia e independencia alimentaria (debido a la posibilidad de criar animales y producir diferentes cultivos) y a su contribución al medio ambiente y futuras generaciones. Por lo que se infiere que en el aspecto social los proyectos y programas son una alternativa para reducir la pobreza, dinamizar economías locales, lograr la seguridad alimentaria y proteger el medio ambiente.

\section{Literatura Citada}

Atkinson, R y Flint, J. 2001. Accessing Hidden and Hard-toReach Populations: Snowball Research Strategies. Social Research UPDATE 1:33-4.

Charles, M. 2007.El impacto de las remesas en los hogares rurales de Honduras. Zamorano, Honduras, $38 \mathrm{p}$.

Chavez, O. y Chafla, M. 2009. Plan de negocios para la exportación de cacao (Theobroma cacao) orgánico al mercado europeo, producido bajo un sistema agroforestal en Catacamas, Honduras. Zamorano, Honduras, $106 \mathrm{p}$.

FHIA (Fundación Hondureña de Investigación Agrícola). 2015a. Atlántida sigue fortaleciendo la producción de cacao en Honduras. Noticias de la FHIA, Octubre 15, No 97 , Honduras, $3 \mathrm{p}$.

FHIA (Fundación Hondureña de Investigación Agrícola). 2015b. Proyecto promoción de sistemas agroforestales de alto valor con cacao en Honduras. La Lima, Cortés, Honduras, $51 \mathrm{p}$.

Fromm, I. 2014. Organic chocolate for the Swiss market: Contract farming in the cocoa sector in Honduras. Da Silva C, Rankin M (eds).Contract Farming for Inclusive Market Access. FAO, Roma.129-141.
Girade, C. 2013. APROSACAO project Olancho region, Honduras, Progress report. Pur Projet. $55 \mathrm{p}$.

Hernández, B. 2015. Sinergias entre adaptación y mitigación al cambio climático en Honduras. Escuela Agrícola Panamericana, Zamorano, Honduras, $35 \mathrm{p}$.

Howard, F., Merida, M. 2014. El taladrador de las meliáceas, Hypsipyla grandella (Zeller) (Insecta: Lepidoptera: Pyralidae: Phycitinae). Entomology and Nematology 1:1-8.

Martínez, C. 2011. Determinación del aporte de los sistemas agroforestales con cacao a las familias productoras, en cinco municipios en los departamentos de Cortés y Yoro, Honduras. ESNACIFOR (Escuela Nacional de Ciencias Forestales). Siguatepeque, Honduras. 96 p.

Murillo, A. 2011. Estimaciones de la deforestación en Honduras. Instituto Nacional de Conservación y Desarrollo Forestal, Áreas Protegidas y Vida Silvestre (ICF). Tegucigalpa, Honduras.18 p.

Romero, R. 2014. Uso actual y oferta de tecnologías sostenibles en las cadenas de valor del cacao en Honduras para mejorar la seguridad alimentaria. Centro Agronómico Tropical de Investigación y Enseñanza (CATIE), Agencia de los Estados Unidos para el Desarrollo Internacional (USAID). Honduras. 48 p.

Sánchez, J., Aguilar, H., Dubón A., Woda, C., Arita, E., Duran, E., Sánchez, W., Medrano, G., Maldonado, L., Merlo, M. 2015. Garantizando una alta calidad en el cacao orgánico a lo largo de la cadena de valor en Honduras. Informa final. FHIA (Fundación Hondureña de Investigación Agrícola). La Lima, Cortés, Honduras. 36 p.

Servicio Meteorológico Nacional Honduras. 2015. Boletín climático para Honduras 01 al 10 de septiembre 2015. Honduras. $8 \mathrm{p}$.

Vallejo, M. 2011. Evaluación preliminar sobre las causas de deforestación y degradación de bosques en Honduras (informa final). Consultoría en legislación y gestión ambiental (ECOJURIS), Tegucigalpa. 142 p.

Recibido para publicación el 6 de diciembre del 2016.

Aceptado para publicación el 1 de agosto del 2017.

Publicado el 12 de febrero del 2018. 